\title{
El estado de la investigación en ciencia política sobre América Latina
}

\author{
The state of political science research on Latin America
}

\author{
SANTIAGO BASABE-SERRANO \\ Facultad Latinoamericana de Ciencias Sociales (FLACSO), Ecuador \\ SERGIO HUERTAS-HERNÁNDEZ \\ Facultad Latinoamericana de Ciencias Sociales (FLACSO), Ecuador
}

\section{Cómo citar/Citation}

Basabe-Serrano, S. y Huertas-Hernández, S. (2018). El estado de la investigación en ciencia política sobre América Latina. Revista Española de Ciencia Política, 47, 153-170. Doi: https://doi.org/10.21308/recp.47.00

\section{Resumen}

Esta investigación analiza el estado de la investigación producida en ciencia política y cuyo objeto de estudio es América Latina. Para ello, presentamos una base de datos inédita en la que incluimos los artículos publicados en revistas en español y portugués que se encuentran en los índices JCR o Scimago. En primer lugar, los resultados reflejan una disciplina dinámica, plural en términos teóricos, inclinada prioritariamente al estudio de los temas clásicos de la disciplina y con falencias en el orden metodológico. Por otro lado, constatamos que existen muy pocas revistas para la publicación, por lo que urge la creación de revistas especializadas. Además, nuestros hallazgos empíricos dan cuenta de la necesidad de incluir en las agendas de investigación tanto a países menos estudiados como temas que, a pesar de ser relevantes $(v$. g. corrupción), han sido poco explorados. Finalmente, este trabajo plantea la urgencia de fomentar la cooperación institucional.

Palabras clave: ciencia política, América Latina, investigación.

\begin{abstract}
This research analyzes the state of Political Science research with a focus on Latin America. To this aim, an unpublished database is presented containing articles published in Spanish and Portuguese journals that are included in JCR or Scimago indexes. First of all, the results show a discipline which is dynamic, theoretically plural, mainly inclined to the study of classic issues within the discipline, and with some methodological failures. On the other hand, it is confirmed the
\end{abstract}


existence of very few journals where to publish, so the creation of specialized ones seems urgent. Furthermore, our empirical findings point out to the necessity of including both under-researched countries and relevant yet underexplored topics (e.g. corruption) in the research agenda. Finally, this work raises the urgency of promoting cooperation at institutional level.

Keywords: political science, Latin America, research.

\section{INTRODUCCIÓN}

Durante las últimas décadas la ciencia política ha crecido exponencialmente en América Latina. A pesar de que el desarrollo de la disciplina es aún asimétrico en los distintos países, en general se observa una mejoría tanto en el plano de la docencia como en el de la investigación ${ }^{1}$. La oferta académica de programas de pregrado y posgrado ha crecido y al mismo tiempo la investigación se ha expandido en cuanto a temáticas estudiadas, países analizados y metodologías utilizadas para el abordaje empírico (Altman, 2012). Adicionalmente, los congresos de las asociaciones nacionales son cada vez más regulares y la Asociación Latinoamericana de Ciencia Política (ALACIP) se ha constituido en el espacio inequívoco de encuentro e intercambio entre estudiantes, profesionales e investigadores de la región.

De las distintas aristas que permiten observar el desarrollo de la ciencia política cuyo objeto de estudio es América Latina, nos concentramos en la investigación científica y específicamente en los trabajos divulgados a través de revistas especializadas publicadas en español y portugués. Identificamos los principales temas y países estudiados, las perspectivas teóricas a las que se suele recurrir y los enfoques metodológicos privilegiados por los autores. Adicionalmente, junto a observar la presencia de mujeres en la producción académica de la región, analizamos tanto la procedencia institucional de los investigadores como el grado de coautoría existente. Finalmente, proponemos algunas ideas de política investigativa que podrían ayudar a la formación de una agenda de trabajo que permita consolidar la ciencia política en la región.

El artículo se estructura alrededor de siete apartados. En el primero detallamos las decisiones metodológicas asumidas para la selección de las revistas incorporadas al análisis. En el segundo apartado discutimos los principales temas estudiados en la región. En el tercero abordamos los enfoques teóricos prioritariamente utilizados. La cuarta sección estudia los principales componentes metodológicos de los artículos

1. Para una revisión histórica de las trayectorias asumidas por la ciencia política en América Latina se puede recurrir a Bulcourf et al., (2015) o Barrientos (2013). Análisis por países se encuentran en los volúmenes 35 (1) de 2015 y 25 (1) de 2005 de la Revista de Ciencia Política. Altman (2012) también plantea un interesante estudio sobre los avances de la ciencia política en América Latina. Sin embargo, dado que el criterio de selección que utiliza Altman está dado en función de los departamentos especializados existentes al interior de las universidades, sus conclusiones se orientan en otro sentido al que este artículo propone. 
analizados. La quinta analiza los países mayoritariamente estudiados, mientras la sexta discute quiénes son los autores de los trabajos, su procedencia institucional y el grado de coautoría existente. La última sección presenta conclusiones y algunas ideas de cara a una agenda de políticas de investigación que fortalezcan la ciencia política latinoamericana.

\section{APUNTES METODOLÓGICOS Y PRINCIPALES REVISTAS}

Para el análisis consideramos los artículos que, desde la ciencia política, presentan resultados de investigación sobre América Latina, escritos en español o portugués, y publicados en revistas especializadas incluidas en los índices Journal Citation Report (JCR) o Scimago. Una ventaja de estudiar las revistas mencionadas es que todas son de libre acceso. Observar solamente resultados de investigación excluye por tanto, los manuscritos que se centran en análisis de coyuntura y que suelen ser publicados por invitación de los editores de las revistas ${ }^{2}$. Excluimos también las notas de investigación y las reseñas bibliográficas. Además, dejamos fuera los trabajos claramente ubicados en la subdisciplina de las relaciones internacionales y aquellos que son traducciones de trabajos previamente publicados en otros idiomas. Desde el punto de vista temporal, nuestra investigación incluyó solo las publicaciones realizadas entre 2011 y 2016. Pese a esta limitación temporal, entendemos que el desempeño de los últimos seis años en la producción académica de la ciencia política sobre América Latina permite tener una idea general del estado de la región en este tema.

Como mencionamos, para la observación nos restringimos a aquellas revistas que están incluidas en los dos índices mencionados, por lo que existiría una presunción objetiva de que son publicaciones periódicas, provistas de doble arbitraje ciego y con parámetros de selección más rigurosos. Dado que la ciencia política llegó a América Latina de forma tardía en comparación con Estados Unidos o Europa, incluir solamente revistas de la disciplina restringía demasiado el número de trabajos para analizar. Por ello, decidimos añadir también revistas de estudios de área o regionales $(v \cdot g$. América Latina Hoy) y otras genéricas de ciencias sociales pero que incluyen trabajos en ciencia política ( $v . g$. Revista de Estudios Sociales). En total valoramos 22 revistas y dentro de ellas identificamos 531 artículos ( $\mathrm{n}=531)$.

Para la selección de artículos analizamos en primer lugar el resumen. Los manuscritos referidos a temas diferentes de los considerados $(v . g$. estudios culturales o literarios) fueron excluidos. El gráfico 1 presenta el porcentaje de trabajos publicados durante el período 2011-2016 en las revistas consideradas para en el análisis. En el anexo 1 incluimos el país en el que está anclada la revista y el grado de impacto en cuartiles a partir de la información reportada por Scimago.

2. Un ejemplo de este caso es el Anuario Político de América Latina, exitosamente publicado por la Revista de Ciencia Politica de la Pontificia Universidad Católica de Chile. 
Gráfico I.

Publicaciones EN CIENCIA POLÍtica POR REVISTAS, 20I I-20I6

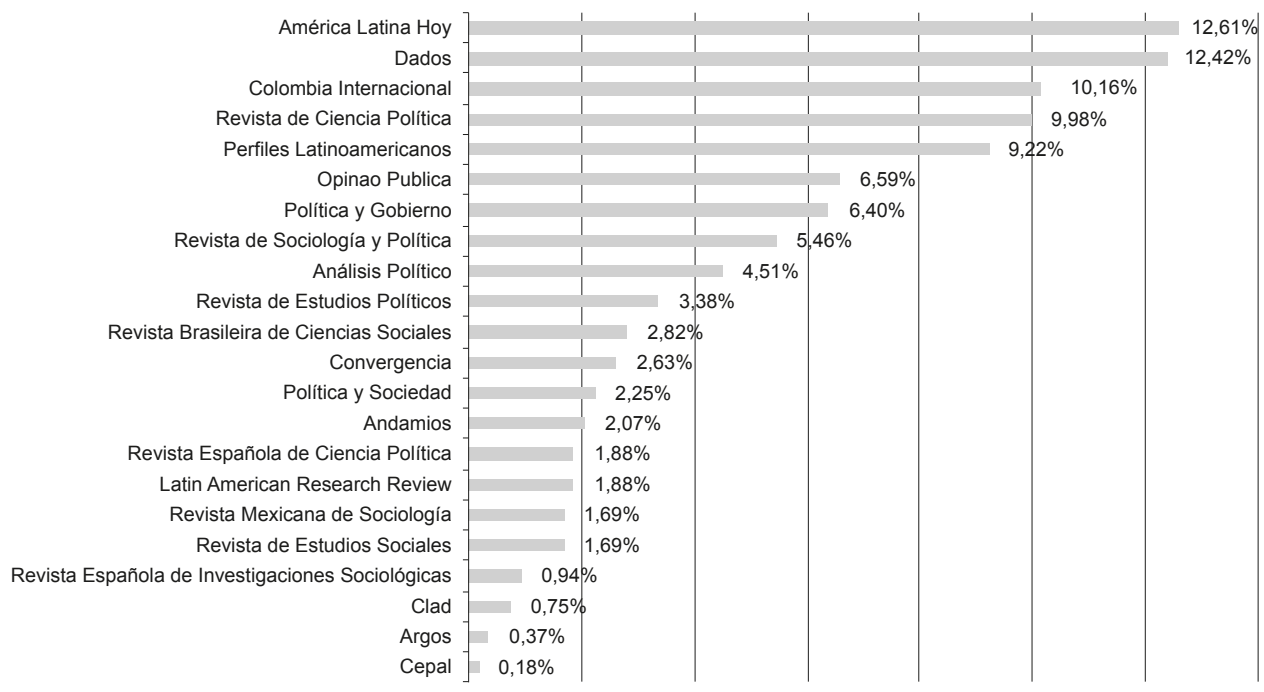

Fuente: elaboración propia a partir de revistas incluidas en JCR y Scimago.

Como se observa, más de la mitad de los artículos publicados se concentran en cinco revistas. De ellas, solamente la Revista de Ciencia Política es una publicación especializada en la materia, mientras que las restantes son de ciencias sociales o de estudios regionales, aunque con cierto énfasis hacia la ciencia política ${ }^{3}$. La otra revista referencial de la disciplina, Política y Gobierno, publica menos a artículos, aunque esto podría obedecer a que se divulga de forma semestral y no trimestral o cuatrimestral como otras publicaciones. En cuanto a la ubicación geográfica en la que se encuentran ancladas las revistas, España, Brasil y México son los países en los que existe un mayor número de publicaciones (ver anexo 1). Es llamativa tanto la ausencia de revistas argentinas en los índices JCR o Scimago como el rol trascendental que viene adquiriendo la revista Colombia Internacional dentro de las publicaciones en ciencia política.

\section{TEMAS ESTUDIADOS}

Para identificar los temas estudiados elaboramos una taxonomía que busca ser lo más inclusiva posible y que se refleja en el gráfico 2. Para el efecto, observamos las

3. El hecho que los editores de América Latina Hoy, Colombia Internacional y Perfiles Latinoamericanos sean politólogos puede haber incidido en que la orientación de los trabajos allí publicados se haya concentrado en alguna medida en la ciencia política. 
temáticas consideradas como ejes para la presentación de ponencias o mesas de trabajo en los congresos internacionales de ciencia política. En ese aspecto, nuestro punto de partida fueron los eventos organizados tanto por la Asociación Latinoamericana de Ciencia Política (AECIP) como también, en la parte pertinente, por la Asociación de Estudios Latinoamericanos (LASA). Posteriormente agregamos algunas categorías temáticas extraídas de congresos nacionales de ciencia política, como el organizado por la Sociedad Argentina de Análisis Político (SAAP) o el que auspicia la Asociación Brasileña de Ciencia Política (ABCP). Por la naturaleza heurística de la taxonomía propuesta, hay temas más analíticos y otros que se encuentran en un mayor nivel de agregación. Sin embargo, hay que apuntar que en ningún caso un artículo aparece incluido en más de una temática, evitando de esa manera problemas metodológicos de sobre o subestimación de los valores constantes en el gráfico 2.

Dentro de las temáticas más estudiados se encuentran los partidos políticos, los procesos electorales y la democracia. En conjunto, estos tres ejes abarcan casi la tercera parte de la muestra analizada y dan cuenta de la preferencia de los investigadores por los temas más clásicos de la disciplina. Dentro de los estudios sobre partidos encontramos análisis endógenos de las estructuras partidistas como también aquellos que observan la interacción respecto a otros partidos y al conjunto del sistema político. En el caso de los trabajos sobre elecciones no solo hay atención a los procesos electorales como tales, sino también al comportamiento de los electores y a la influencia de las reglas electorales sobre los resultados políticos. La preeminencia otorgada al estudio de partidos y elecciones tiene un símil en el trabajo realizado por Boncourt (2008) para Europa. En cuanto a los estudios sobre democracia, la medición de la calidad del régimen, sus rendimientos e interacciones respecto a actores sociales y económicos son las facetas más exploradas. No obstante, durante los últimos ańos se han incrementado las investigaciones sobre democracia participativa, democracia directa y e-democracy.

Un segundo conjunto de temas incluye el estudio de la estructura, funcionamiento e interacciones de las legislaturas, las cortes de justicia y el poder ejecutivo. Los estudios legislativos enfatizan el comportamiento de los diputados en cuanto al voto y la formación de coaliciones frente al presidente, mientras que los relacionados con el campo conocido como "políticas judiciales» — judicial politics — se centran en la relación de los jueces respecto a los políticos en temas como la independencia judicial o la judicialización de la política desde las cortes constitucionales. En cuanto a las investigaciones sobre presidencialismo, se observa un incremento en el estudio tanto de los gabinetes ministeriales como respecto al funcionamiento de las casas presidenciales. No obstante, la pregunta sobre cuán presidencialistas son los países de la región sigue siendo el eje de la investigación en este campo.

Por otro lado, están los trabajos sobre teoría política y políticas públicas. En el primer caso, la principal estrategia utilizada por los investigadores radica en el estudio de determinados autores clásicos del pensamiento político y su posible interpretación a la luz de los eventos contemporáneos. En el segundo caso, los trabajos se refieren esencialmente a la gobernanza en sus distintos niveles y a la formulación y evaluación de 
políticas públicas. En términos empíricos, se trata de investigaciones aplicadas a diferentes sectores como el de hidrocarburos, salud pública o educación superior. Un hallazgo interesante es que los estudios de teoría política y, fundamentalmente, los de políticas públicas, se encuentran bien situados dentro de las preferencias de los investigadores a pesar de no ser parte de los temas clásicamente abordados por la ciencia política. El tercer grupo de temas incluye trabajos sobre la presencia de mujeres en diferentes arenas de toma de decisión política, las transformaciones del Estado, los estudios de opinión pública y también las investigaciones relacionadas con las élites políticas.

Junto a los temas ya mencionados, el gráfico 2 describe otros tópicos que, por el contrario, han sido marginalmente analizados por la ciencia política latinoamericana a pesar de su trascendencia. Entre ellos se encuentra el estudio de la corrupción, una de las grandes problemáticas de la región, y que a pesar de ello registra apenas el $1,08 \%$ del total de artículos analizados. Un argumento parecido aplicaría para el caso de los estudios relacionados con la confianza ciudadana, tanto en el régimen democrático como inter pares $(1,26 \%)$, el clientelismo político $(0,90 \%)$ o los trabajos en los que se vinculan las interacciones entre variables económicas y políticas como elemento clave para la comprensión de distintos fenómenos sociales $(0,90 \%)$. Finalmente, la evidencia empírica ofrecida da cuenta de la escasez de trabajos que discuten cuestiones metodológicas $(0,72 \%)$.

GrÁfICO 2.

TeMAS ESTUdiados EN LA CIENCIA POLÍTICA LATINOAMERICANA, 20 I I-20I 6

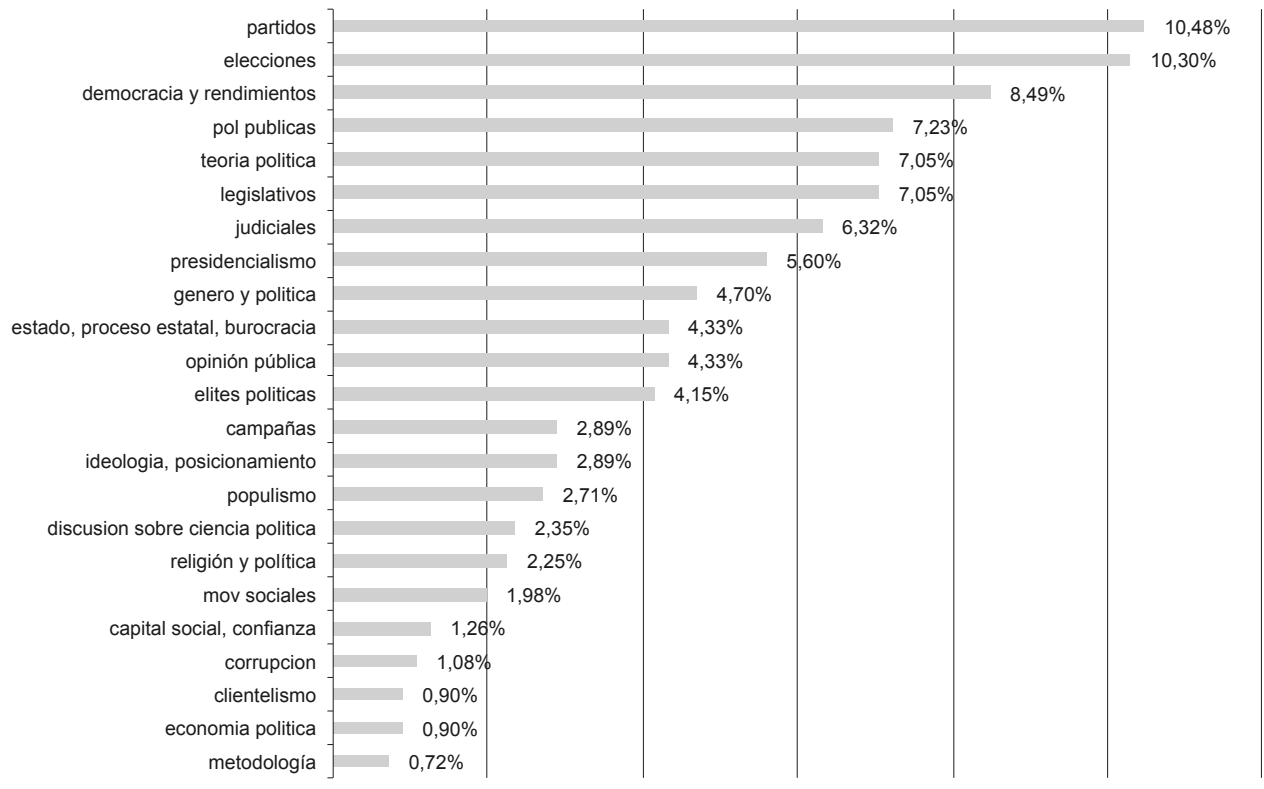

Fuente: elaboración propia a partir de revistas incluidas en JCR y Scimago. 


\section{ENFOQUES TEÓRICOS}

En cuanto a las teorías más utilizadas por los investigadores, las preferencias están entre los enfoques sistémicos $(29,56 \%)$, neoinstitucionales $(27,60 \%)$ y del elector racional $(25,65 \%)$. Una porción más reducida de artículos asume teorías propias de los estudios de cultura política (11,08\%), mientras que el 6,08 \% incluye otras perspectivas teóricas. Dentro del enfoque prioritariamente abordado se encuentran tanto los trabajos derivados del estudio de los sistemas políticos y específicamente del régimen democrático como aquellos interesados en observar los partidos en su mutua interacción y respecto a los sistemas electorales. La extensa obra de Sartori (2005), Easton (1965; 1999; 1997) o Rapoport (1997) y la influencia de la vertiente europea en la formación académica de las primeras generaciones de politólogos de América Latina, explicarían el espacio aún influyente de dichas perspectivas teóricas ${ }^{4}$. A diferencia de la investigación proveniente de Estados Unidos, donde a partir de la década de los sesenta la tradición del elector racional y posteriormente la neoinstitucionalista relegaron casi por completo a los estudios sistémicos, dicha perspectiva teórica constituye aún un rasgo distintivo de la ciencia política latinoamericana (Norris, 1997). El gráfico 3 resume las tradiciones teóricas utilizadas en los trabajos incluidos en el análisis.

GrÁfico 3.

PrinCIPALES ENFOQUeS TEÓRICOS

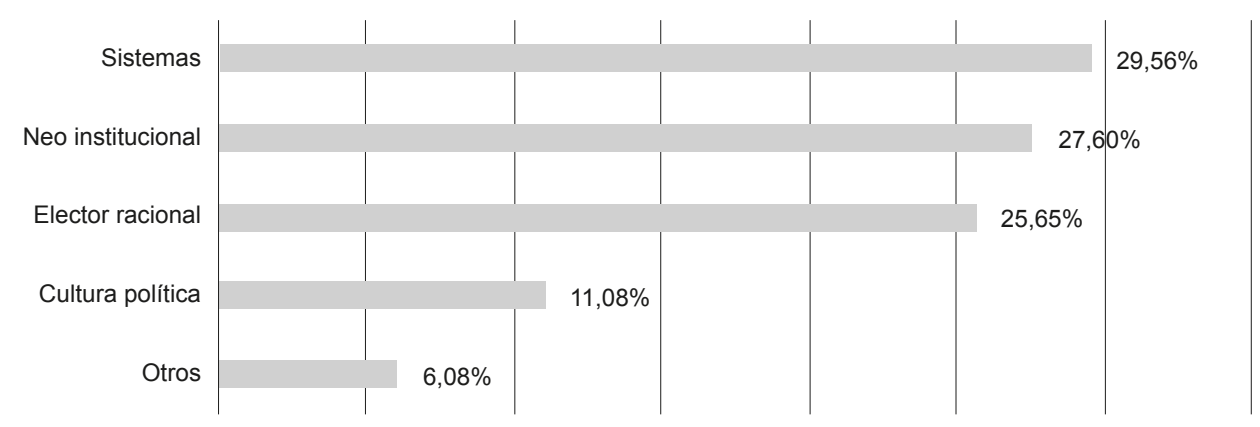

Fuente: elaboración propia a partir de revistas incluidas en JCR y Scimago.

Respecto a los artículos escritos desde el enfoque neoinstitucionalista (March y Olsen, 2008), no existe una preferencia específica hacia alguna de las distintas perspectivas que se inscriben en dicha tradición. De hecho, hallamos trabajos fundamentados tanto en el institucionalismo histórico como en el analítico o el de actores racionales. Aunque en menor medida, también se reportaron artículos que corresponderían a lo

4. Una de las obras clásicas de Sartori (2005) y que generó gran influencia en los estudios políticos sobre América Latina fue «Partidos y sistemas de partidos». En el caso de Easton (1999) quizás la obra que capturó mayor atención en esa región fue «Esquema para el análisis político». 
que Peters (2003) denomina institucionalismo empírico. En cuanto a las investigaciones ancladas en la tradición del elector racional (Riker, 1962) —dentro de las que se incluyeron los trabajos que se sustentan en la teoría de juegos — son menos frecuentes las que recurren a modelos formales y análisis cuantitativo. En la mayoría de los casos los artículos que utilizan este enfoque teórico asumen la perspectiva de la racionalidad limitada (Elster, 1988) y en lo metodológico recurren a narrativas analíticas (Bates et. al., 1998). En resumen, desde el punto de vista teórico, la ciencia política latinoamericana es bastante plural, por lo que no existe un paradigma imperante.

En cuanto al nivel de acercamiento a la realidad, aunque la mayoría de los trabajos analizados son deductivos $(61,20 \%)$, una porción importante plantea perspectivas inductivas $(38,80 \%)^{5}$. Si bien no desconocemos que, en determinados contextos y sobre todo cuando la literatura especializada es residual, los trabajos que parten de la evidencia empírica son necesarios, la posibilidad de generalizar los hallazgos surge de la confrontación de conjeturas razonadas con evidencia empírica. Conforme lo discutimos en el siguiente párrafo, a la presencia de trabajos inductivos se debe agregar que un porcentaje estimable de ellos son estudios de caso. De hecho, del total de artículos que presentan evidencia empírica, el 34,18 \% asumen una perspectiva inductiva sobre una sola unidad de análisis.

\section{COMPONENTES METODOLÓGICOS}

Para valorar la propuesta metodológica hallada en los artículos seleccionados, consideramos varias dimensiones de análisis. En primer lugar, observamos los métodos a los que prioritariamente recurren los investigadores. Un primer hallazgo da cuenta de la ausencia de experimentos — naturales, de laboratorio o vía encuestas de opinión pública - y la total preferencia por métodos observacionales. Esta deficiencia en cuanto al uso de experimentos da cuenta de la necesidad de incorporar esta temática a la discusión metodológica de la ciencia política latinoamericana. Dentro de los métodos observacionales o cuasi experimentales, el 66,29\% de los trabajos analizados se corresponden con estudios de caso, mientras que solamente el $33,71 \%$ recurren al método comparado. Incluimos en esta categoría tanto a las investigaciones con pocas unidades de análisis como a aquéllas con $n$ grande. Si partimos de la idea de que la comparación es el método que de mejor forma nos permite acercarnos a resultados con menor sesgo y mayor control sobre las variables, este hallazgo es llamativo, pues refleja la necesidad de incentivar este tipo de diseńo de investigación en América Latina. La referida prioridad otorgada a los estudios de caso en esta región es un fenómeno similar al observado hasta la década de los noventa en la ciencia política alemana (Pehl, 2012).

Dentro de los artículos que recurren a la comparación, el 81,9\% lo hace entre países y apenas el 18,1 \% se concentra en el análisis subnacional. Puesto que la comparación de

5. En el citado trabajo de Munck y Snyder (2007), los trabajos con un enfoque inductivo alcanzan el $55,7 \%$. 
unidades de análisis dentro de un país aumenta la precisión y especificidad de los diseños de investigación, dado el control que se mantiene sobre variables que podrían cambiar al observar varios países - $v$. $g$. diseño institucional—o por posibles sesgos de equivalencia, en esta parte señalamos la necesidad de prestar mayor atención a este tipo de perspectivas. En cuanto al espacio temporal utilizado para el análisis, el 61,93\% de los trabajos observados se desarrollan desde un enfoque sincrónico y solamente el 38,07 \% utilizan una perspectiva diacrónica. En este aspecto, es indispensable fortalecer las investigaciones que observen procesos de mediano y largo plazo, pues esto incrementa la capacidad de comprensión de los fenómenos políticos a partir de la incorporación de variables contextuales y de la comparación de diferentes períodos temporales.

En cuanto al tipo de herramientas utilizadas para el análisis, un poco más de la mitad de los trabajos observados recurren a técnicas cualitativas como las narrativas históricas, el rastreo de procesos o las cadenas causales (59,35 \%). La mayoritaria presencia de trabajos de orden cualitativo también es un rasgo distintivo del estado de la investigación en Francia (Billordo, 2005). En cuanto a las técnicas cuantitativas, alcanzan un estimable $37,87 \%$ de los artículos, aunque con mayor énfasis en las herramientas de estadística descriptiva respecto a las de naturaleza inferencial ${ }^{6}$. Puesto que la formación metodológica en técnicas cuantitativas no ha sido imperante en América Latina, los hallazgos descritos dan cuenta de que el interés por este tipo de herramientas va en aumento. Finalmente, los artículos que apelan a métodos mixtos son minoritarios $(2,77 \%)$ y reflejan la necesidad de incentivar en la región el uso interactivo de estrategias cuantitativas y cualitativas alrededor de un mismo proyecto de investigación (Lieberman, 2005). El gráfico 4 describe los temas antes discutidos.

GráfICO 4 .

Aspectos Metodológicos De LOS ARTí́culos ANALIZAdos

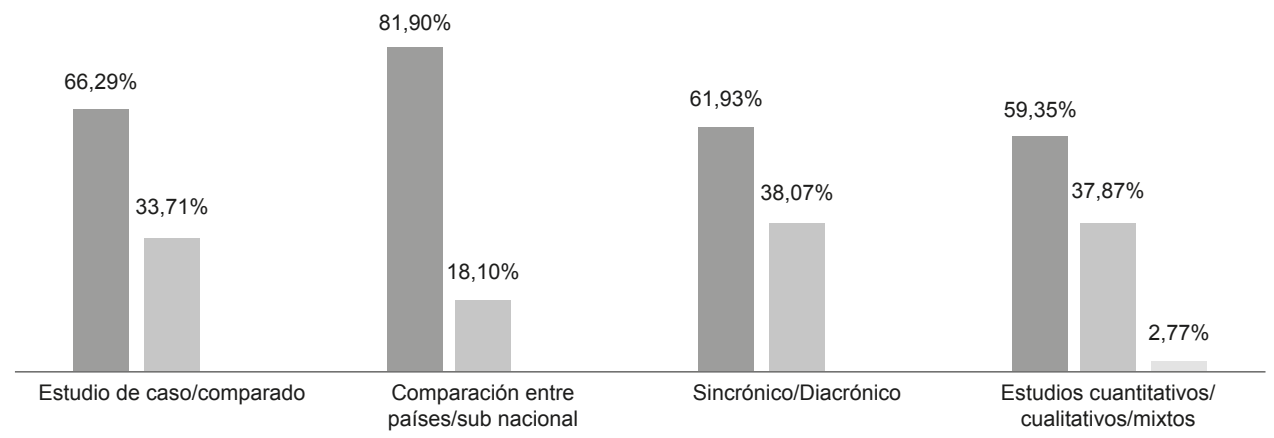

Fuente: elaboración propia a partir de revistas incluidas en JCR y Scimago.

6. Boncourt (2008) da cuenta de una producción selectiva de trabajos cuantitativos en función de la revista estudiada. Así, encuentra que en la revista European Journal of Political Research las investigaciones cuantitativas ascienden al 55 \%, mientras que en Revue Francaise de Science Politique apenas alcanzan el $6 \%$. 


\section{PAÍSES MÁS ESTUDIADOS}

Antes de analizar los países que con mayor frecuencia han sido seleccionados como unidades de análisis, hemos tomado algunas decisiones metodológicas adicionales a las ya citadas. En primer lugar, y únicamente para el análisis que sigue, descartamos tanto los trabajos de teoría política como aquellos en los que no existía una constatación empírica. Adicionalmente, dejamos de lado las pocas investigaciones de $n$ grande pues allí cada país constituye solamente una observación. Con dichos presupuestos, y al igual que en investigaciones focalizadas en el estudio de legislaturas (Alemán, 2013), cortes de justicia (Kapiszewski y Taylor, 2008) o burocracias (Polga-Hecimovich y Trelles, 2016), Brasil es el país que mayor atención recibe de los politólogos de la región (31,52\%). A una distancia considerable se encuentran México (16\%), Colombia $(10,34 \%)$, Chile $(10,09 \%)$ y Argentina $(9,35 \%)$. No obstante las brechas entre Brasil y los demás países citados, este conjunto de unidades de análisis coincide también con las áreas geográficas en las que la ciencia política latinoamericana ha logrado mayor desarrollo en el campo docente.

Gráfico 5.

PAÍsES MÁs ESTUdiados

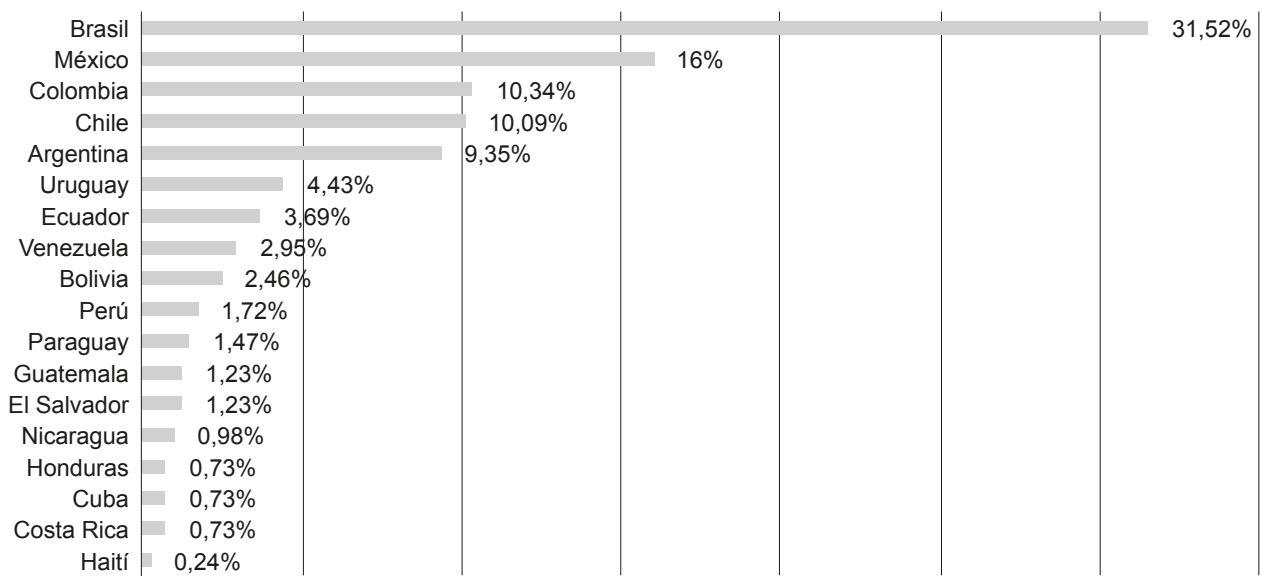

Fuente: elaboración propia a partir de revistas incluidas en JCR y Scimago.

Un segundo grupo incluye a Uruguay (4,43\%), Ecuador (3,69 \%), Venezuela $(2,95 \%)$ y Bolivia (2,46\%). Este hallazgo empírico es llamativo, pues revela un mayor interés por países como Ecuador y Bolivia, que hasta hace pocos años atrás habían sido poco estudiados. Desde luego, el hecho de que sean considerados como unidades de análisis no implica necesariamente la existencia de un mayor número de investigadores ecuatorianos y bolivianos, pero al menos es un indicio respecto al desarrollo que podría adquirir la disciplina en ambos países. La presencia de asociaciones nacionales de ciencia política tanto en Ecuador como en Bolivia constituye otro elemento de 
juicio que amplía las expectativas ya citadas. Conforme consta en el gráfico 5, el resto de países de la región tienen menos del $2 \%$ de trabajos en los que se los considera como unidades de análisis. Entre ellos, sobresalen Perú y Costa Rica, pues ambos han recibido una mayor atención en mediciones más específicas, como la de Alemán (2013) respecto al estado de la investigación en legislaturas.

\section{AUTORES E INSTITUCIONES}

En esta parte observamos algunas dimensiones de análisis relacionadas tanto con los autores de los artículos como con su pertenencia institucional. Para la codificación consideramos algunas variables. En primer lugar, se capturó el sexo del autor del artículo. Posteriormente, consideramos una variable dicotómica que refleja si el manuscrito ha sido escrito por uno o más autores. En cuanto a la pertenencia institucional, observamos el espacio geográfico en el que está la universidad o centro de investigación en el que trabaja el investigador. Por tanto, no se capturó información sobre la nacionalidad de los autores. En los casos en los que hay coautoría y los investigadores pertenecen a instituciones geográficamente distintas elaboramos categorías de análisis específicas, tal cual consta en el gráfico 6 .

En cuanto al sexo de la autoría del artículo, la información recopilada revela que cerca de las dos terceras partes de los trabajos son escritos por hombres $(60,26 \%)$, mientras que apenas el $22,97 \%$ corresponden a mujeres. Esta asimetría se correlaciona con la deficitaria presencia de politólogas latinoamericanas y, a la vez, da cuenta de la reproducción en el mundo académico de las históricas desigualdades existentes en la región. La autoría del restante 16,76 \% de artículos corresponde al trabajo conjunto de hombres y mujeres. Un dato llamativo es que los estudios desde la ciencia política relacionados con la mujer y su incidencia sobre diferentes arenas de toma de decisión pública no son el campo temático que mayor interés suscita entre las politólogas latinoamericanas. Otro dato interesante es que la asimetría entre producción de hombres y mujeres en Latinoamérica es menor que la reportada por Munck y Snyder (2007) para el caso de Estados Unidos?

Por otro lado, para valorar en qué medida se ha generado una comunidad académica alrededor de la ciencia política latinoamericana, observamos las coautorías constantes en los artículos analizados. A diferencia de lo que señalan Pehl (2012) o McDermott y Hatemi (2010) en el sentido de que las investigaciones de dos o más autores dentro de la ciencia política alemana y norteamericana se han incrementado, en América Latina los artículos escritos por un solo investigador siguen siendo

7. El trabajo citado recurre al análisis de tres de las más importantes revistas en habla inglesa (Comparative Politics, Comparative Political Studies y World Politics). En función de dicho criterio de selección, la asimetría entre la producción de hombres $(74,3 \%)$ y mujeres $(18,5 \%)$ es mayor que en América Latina (Munck y Snyder, 2007). La producción mixta alcanza el 7,2 \%. 
mayoritarios $(62,17 \%)^{8}$. La producción investigativa de dos autores alcanza apenas a la cuarta parte del universo de trabajos analizados $(25,98 \%)$ y solamente el $11,28 \%$ corresponde a artículos escritos por tres o más autores.

En cuanto a la ubicación geográfica de las instituciones donde se genera la producción académica, e independientemente de la nacionalidad de los investigadores, más de las tres cuartas partes de los artículos provienen de universidades o centros de estudios ubicados en América Latina (76,08 \%). En Europa se produce el 13,18 \% de los trabajos analizados y apenas el 3,76\% se ubica en instituciones ubicadas en Estados Unidos. La débil presencia de artículos escritos en Estados Unidos resulta contraintuitiva por al menos dos razones. En primer lugar, porque los politólogos latinoamericanos residentes fuera de la región se asientan prioritariamente en ese país y se podría pensar que al menos parte de su producción se divulgaría en revistas en español o portugués. En segundo lugar, porque los centros de investigación y universidades con departamentos de Ciencia Política interesados en América Latina están en Estados Unidos y ello haría suponer que, en alguna medida, los artículos allí generados se difundirían en revistas de la región estudiada. Además, resulta llamativo el número de artículos provenientes de investigadores residentes en Europa, pues dadas las razones ya mencionadas, era esperable que la cantidad de trabajos generados en dicho continente fuese comparativamente menor que los procedentes de Estados Unidos. El gráfico 6 describe la discusión precedente.

Gráfico 6.

UBiCACiÓN GEOGRÁFICA DE LAS INSTITUCIONES EN LAS QUE SE GENERA LA PRODUCCiÓN ACADÉMICA

\begin{tabular}{ccccccccc}
\hline & & & & & & \\
\hline \\
\hline
\end{tabular}

Fuente: elaboración propia a partir de revistas incluidas en JCR y Scimago.

8. Hay que considerar que si bien McDermott y Hatemi (2010) tienen un período de estudio que empieza en la década de los setenta del siglo pasado, su observación se limita a la revista American Political Science Review. 
Las distancia entre la producción académica generada en América Latina respecto a la procedente de Estados Unidos se torna más pronunciada si se agrega que el tipo de lector de las publicaciones en inglés respecto a las de español o portugués es marcadamente diferente. Lo dicho se sustenta en al menos dos razones. Por un lado, y a diferencia de las revistas en español o portugués, la gran mayoría de publicaciones en inglés no son de libre acceso. Este es un hecho clave, pues muchas universidades latinoamericanas carecen de fondos para pagar suscripciones, tanto a revistas como a bases de datos $(v . g$. JSTOR o SAGE) que permitan a sus estudiantes acceder gratuitamente a la información. Por otro lado, el bajo nivel de lectura en inglés de los latinoamericanos constituye una barrera adicional para acceder al conocimiento escrito en ese idioma.

Sobre este último aspecto, el informe de Napoleón Katsos, socio directivo del Centro de Investigación de Inglés y Lingüística Aplicada de la Universidad de Cambridge, evidencia que ninguno de los trece países latinoamericanos incluidos en su estudio tiene nivel «muy alto» o «alto» de inglés. Los países mejor situados son Argentina y México, y apenas se ubican en la categoría de nivel «medio». Costa Rica, Guatemala, El Salvador y Brasil están en nivel «bajo», mientras que Dominicana, Perú, Chile, Ecuador, Venezuela, Panamá y Colombia son colocados en un nivel de inglés «muy bajo»". Las razones mencionadas dan cuenta, por tanto, de que existe un problema estructural que debe ser solventado para a establecer más espacios de diálogo entre la investigación norteamericana y la de América Latina. Por tanto, es crucial el acceso a las publicaciones en inglés y también la mejora del nivel de comprensión de ese idioma.

\section{CONCLUSIONES Y AGENDA DE POLÍTICAS PARA LA INVESTIGACIÓN EN CIENCIA POLÍTICA DESDE AMÉRICA LATINA}

Este artículo analiza el estado de la investigación generada por la ciencia política latinoamericana a lo largo del período 2011-2016. A partir de la valoración efectuada, podemos identificar tanto los avances como los desafíos que se presentan a la disciplina a corto y mediano plazo. En primer lugar, la variedad de temáticas abordadas, la recurrencia a diferentes perspectivas teóricas y la cada vez mayor presencia de evidencia empírica que acompaña a los trabajos dan cuenta de avances importantes en la ciencia política de la región. A pesar de que la aparición de la disciplina en América Latina es tardía, el proceso de institucionalización en el plano de la docencia, investigación y generación de comunidad académica es sostenido, aunque puede ser fortalecido desde diferentes áreas.

En el plano de la divulgación, una posibilidad es la creación de revistas generales de ciencia política, por un lado, y especializadas en diferentes subdisciplinas por otro. Salvo la Revista de Ciencia Política y Política y Gobierno, en América Latina no se dispone de más espacios para publicar la investigación sobre la disciplina. Aunque el

9. Se puede acceder a la investigación referida en el link: https://bit.ly/2NxDxbv. 
apoyo de las otras revistas analizadas en este artículo ha sido clave, el hecho de no ser publicaciones especializadas hace que los espacios tengan que ser compartidos con otras disciplinas, lo que genera un problema de incentivos selectivos para unos y otros. En esta misma línea, otro reto que afronta la investigación de la ciencia política latinoamericana tiene que ver con el incremento en la calidad, visibilidad e impacto de la producción científica (Buquet, 2013). Un camino para resolver, al menos parcialmente, las deficiencias anotadas, está en la cooperación interinstitucional o el apoyo de las asociaciones nacionales de ciencia política, desde donde se podrían dar los primeros pasos para el establecimiento de revistas que cubran los parámetros mínimos para ser agregadas a los índices JCR o Scimago.

En cuanto a los temas estudiados, y sin que esto implique dejar de lado los campos más tradicionales de la ciencia política, este artículo ha constatado poca atención hacia temas clave como la corrupción, el clientelismo, la representación política de mujeres o la relación entre desempeños económicos y resultados políticos. En el campo teórico se ha evidenciado que los enfoques sistémicos junto a los neoinstitucionales y del elector racional comparten las preferencias de los investigadores. No obstante, si la prevalencia del enfoque sistémico tiene relación con la conjetura que hemos planteado, en el sentido de que esta orientación corresponde a la formación académica de los primeros politólogos de la región, es de esperar que los próximos años la ciencia política latinoamericana cuente con una perspectiva más analítica. Consideramos que la mayor presencia de enfoques más analíticos, servirán para ganar en precisión en cuanto a los argumentos y a la observación empírica de variables o procesos. Adicionalmente, los datos aportados en este artículo dan cuenta de la presencia marginal de perspectivas teóricas estructuralistas dentro de la ciencia política de la región.

La metodología es el campo que más trabajo requiere y constituye el mayor desafío para la ciencia política de América Latina. Una necesidad imperiosa está en fomentar la investigación comparada y reorientar el estudio de caso con una observación hacia otros estudios en los que existan más unidades de análisis para poder comparar inclusive dentro de un mismo país o entidad (Gerring, 2004) ${ }^{10}$. No obstante, la ausencia relativa de investigación comparada es un fenómeno trasladable, al menos hasta fines de la década de los noventa del siglo pasado, a la ciencia política norteamericana y europea (Norris, 1997). En este plano, incrementar el trabajo en estudios subnacionales puede favorecer el control de variables y la mayor precisión de los diseños de investigación. En cuanto a las técnicas de investigación, las de naturaleza cuantitativa van ganando espacio en la región y eso favorece la diversidad de la disciplina. Por tanto, un desafío adicional está en la inclusión de cursos de metodologías cuantitativas en los programas docentes de la región. Esta política es esencial, aunque debe ser tratada de forma crítica, evitando que la «sofisticación» cuantitativa degenere en situar las técnicas metodológicas por encima de las ideas.

10. No obstante, la ausencia relativa de investigación comparada es un fenómeno trasladable, al menos hasta fines de la década de los noventa del siglo pasado, a la ciencia política norteamericana y europea (Norris, 1997). 
Al igual que en estudios previos, constatamos que Brasil es la unidad de análisis más estudiada de la región, mientras que es muy poco lo que conocemos de los países centroamericanos. El que las revistas tengan mayores incentivos para publicar artículos de investigación sobre países poco estudiados, pues ello afectaría positivamente a su tasa de citaciones, debería motivar a los investigadores a prestar mayor interés respecto a unidades de análisis menos explorados de América Latina. Adicionalmente, fomentar la extracción y producción de información y datos desde las instituciones académicas de los países menos estudiados podría facilitar que investigadores locales e internacionales se acercaran más al estudio de dichas unidades de análisis. No obstante, la desatención que se observa hacia varios países de América Latina se podría explicar por los altos costos de transacción que conlleva obtener evidencia empírica.

Finalmente, la deficitaria presencia de artículos publicados por politólogas y aquellos que son resultado de la cooperación entre universidades y centros de investigación dan cuenta de espacios en los que se debe trabajar intensamente en el futuro inmediato. No obstante, la promoción que han recibido durante los últimos ańos los congresos disciplinarios por países, los grupos de trabajo auspiciados desde diferentes organizaciones y la articulación de ambas actividades alrededor de la Asociación Latinoamericana de Ciencia Política son signos positivos en el sentido de que estas carencias pueden irse superando. Independientemente de lo dicho, la reducción de las asimetrías de diverso orden, el incremento de la confianza interpares y su efecto directo en términos de cooperación son temas estructurales en los que la evidencia empírica que hemos expuesto no es más que el reflejo de un problema social de mayores dimensiones dentro de la región.

\section{Referencias}

Alemán, Eduardo. 2013. «Latin American Legislative Politics: A Survey of Peer-Reviewed Publications in English", Journal of Politics in Latin America, 5 (1): 15-36.

Altman, David. 2012. "Where is Knowdledge Generated? On the Productivity and Impact of the Political Science Department in Latin America», European Political Science, 11 (1): 1-17. Disponible en: https://doi.org/10.1057/eps.2010.82.

Barrientos del Monte, Fernando. 2013. «La Ciencia Política en América Latina. Una breve introducción histórica», Convergencia: Revista de Ciencias Sociales, 20 (61): 105-133.

Bates, Robert H., Avner Greif, Margaret Levi y Jean-Laurent Rosenthal. 1998. Analytic Narratives. Princeton: Princeton University Press.

Billordo, Libia. 2005. «Publishing in French Political Science Journals: an Inventory of Methods and Sub-fields», French Politics 3 (2): 178-186. Disponible en: https:// doi.org/10.1057/palgrave.fp.8200075.

Boncourt, Thibaud. 2008. «Is European Political Science Different from European Political Sciences? A Comparative Study of the European Journal of Political Research, Political Studies and the The Revue francaise de science politique, 
1973-2002», European Political Science 7 (3): 366-381. Disponible en: https://doi. org/10.1057/palgrave.eps.2210185.

Bulcourf, Pablo, Enrique Gutiérrez Márquez y Nelson Cardozo. 2014. «El desarrollo de la ciencia política en Argentina, Brasil y México: construyendo una mirada comparada", Anuario Latinoamericano. Ciencias Politicas y Relaciones Internacionales, 1 (1): 155-184.

Bulcourf, Pablo, Enrique Gutiérrez Márquez y Nelson Cardozo. 2015. «Historia y desarrollo de la Ciencia Política en América Latina: reflexiones sobre la construcción del campo de estudios", Revista de Ciencia Politica, 35 (1): 179-199. Disponible en: https://doi.org/10.4067/S0718-090X2015000100009.

Buquet, Daniel. 2013. Producción e impacto de las ciencias sociales en América Latina. Documento de Trabajo. Buenos Aires: CLACSO.

Easton, David. 1965. A Systems Analysis of Political Life. New York: John Wiley and Sons.

Easton, David. 1997. "Categorías para el análisis sistémico de la política», en David Easton (comp.), Enfoques sobre teoría politica. Buenos Aires: Amorrortu.

Easton, David. 1999. Esquema para el análisis politico. Buenos Aires: Amorrortu.

Elster, Jon. 1988. Uvas amargas: sobre la subversión de la racionalidad. Barcelona: Península, Ideas.

Gerring, John. 2004. "What is a Case Study and What Is Good For?», American Political Science Review, 98 (2): 341-354.

Kapiszewski, Diana y Matthew M. Taylor. 2008. «Doing Courts Justice?: Studying Judicial Politics in Latin America», Perspectives on Politics, 6 (4): 741-767. Disponible en: https://doi.org/10.1017/S1537592708081899.

Lieberman, Evan S. 2005. "Nested Analysis as a Mixed-Method Strategy for Comparative Research", American Political Science Review, 99 (3): 435-452. Disponible en: https://doi.org/10.1017/S0003055405051762.

March, James G. y Johan P. Olsen. 2008. Elaborating the «new institutionalism», en Sarah A. Binder, R. A. W. Rhodes, y Bert A. Rockman (eds.), The Oxford Handbook of Political Institutions. Nueva York: Oxford University Press.

McDermott, Rose y Peter K. Hatemi. 2010. «Emerging Models of Collaboration in Political Science: Changes, Benefits, and Challenges», PS: Political Science and Politics, 43 (1): 49-58. Disponible en: https://doi.org/10.1017/S1049096510990811.

Munck, Gerardo L. y Richard Snyder. 2007. "Who Publishes in Comparative Politics? Studying the World from the United States», Comparative Political Studies, 40 (1): 5-31. Disponible en: https://doi.org/10.1177/0010414006294815.

Norris, Pipa. 1997. "Toward a More Cosmopolitan Political Science?», European Journal of Political Research, 31: 17-34. Disponible en: https://doi.org/10.1111/ j.1475-6765.1997.tb00761.x.

Pehl, Malte. 2012 «The Study of Politics in Germany: A Bibliometric Analysis of Subfields and Methods», European Political Science, 11 (1): 57-70. Disponible en: https://doi.org/10.1057/eps.2011.38. 
Peters, Guy. 2003. El nuevo institucionalismo: teoría institucional en Ciencia Politica. Barcelona: Gedisa.

Polga-Hecimovich, John y Alejandro Trelles. 2016. «The Organizational Consequences of Politics: A Research Agenda for the Study of Bureaucratic Politics in Latin America», Latin American Politics and Society, 58 (4): 56-79. Disponible en: https://doi.org/10.1111/laps.12002.

Rapoport, Anatol. 1997. "Algunos enfoques sistémicos de la teoría política», en David Easton (comp.), Enfoques sobre teoría politica. Buenos Aires: Amorrortu.

Riker, William H. 1962. The theory of political coalitions. New Haven y Londres: Yale University Press.

Sartori, Giovanni. 2005. Partidos y sistemas de partidos. Madrid: Alianza Editorial.

\section{ANEXO}

País EN EL QUe LA REVISTA ESTA ANCLADA Y GRADO DE IMPACto

\begin{tabular}{llcccccc}
\hline \multirow{2}{*}{ Revista } & \multirow{2}{*}{ País } & \multicolumn{5}{c}{ Cuartil (Scimago) } \\
\cline { 2 - 7 } América Latina Hoy & España & - & Q4 & Q3 & Q4 & Q3 & Q3 \\
\hline Dados & Brasil & Q3 & Q1 & Q2 & Q2 & Q2 & Q2 \\
\hline Colombia Internacional & Colombia & - & Q4 & Q4 & Q4 & Q3 & Q3 \\
\hline Revista de Ciencia Política & Chile & Q3 & Q2 & Q2 & Q2 & Q3 & Q2 \\
\hline Perfiles Latinoamericanos & México & Q4 & Q4 & Q4 & Q3 & Q4 & Q4 \\
\hline Opinião Pública & Brasil & Q2 & Q2 & Q2 & Q2 & Q3 & Q2 \\
\hline Política y Gobierno & México & Q3 & Q3 & Q4 & Q3 & Q3 & Q3 \\
\hline $\begin{array}{l}\text { Revista de Sociología y } \\
\text { Política }\end{array}$ & Brasil & Q3 & Q3 & Q3 & Q2 & Q3 & Q2 \\
\hline Análisis Político & Colombia & Q4 & Q3 & Q4 & Q3 & Q3 & Q3 \\
\hline Revista de Estudios Políticos & Espańa & Q2 & Q4 & Q3 & Q3 & Q3 & Q3 \\
\hline $\begin{array}{l}\text { Revista Brasileira de Ciências } \\
\text { Sociais }\end{array}$ & Brasil & Q3 & Q3 & Q2 & Q2 & Q3 & Q2 \\
\hline Convergencia & México & Q4 & Q4 & Q4 & Q4 & Q4 & Q3 \\
\hline Política y Sociedad & Espańa & - & Q4 & Q3 & Q3 & Q3 & Q4 \\
\hline Andamios & México & Q4 & Q4 & Q4 & Q4 & Q4 & Q3 \\
\hline $\begin{array}{l}\text { Revista Española de Ciencia } \\
\text { Política }\end{array}$ & España & - & - & Q4 & Q4 & Q3 & Q4 \\
\hline $\begin{array}{l}\text { Latin American Research } \\
\text { Review }\end{array}$ & Estados Unidos & Q2 & Q2 & Q2 & Q2 & Q2 & Q2 \\
\hline & & & & & & & .1
\end{tabular}




\begin{tabular}{|c|c|c|c|c|c|c|c|}
\hline \multirow{2}{*}{ Revista } & \multirow{2}{*}{ País } & \multicolumn{6}{|c|}{ Cuartil (Scimago) } \\
\hline & & 2011 & 2012 & 2013 & 2014 & 2015 & 2016 \\
\hline $\begin{array}{l}\text { Revista Mexicana de } \\
\text { Sociología }\end{array}$ & México & - & Q4 & Q4 & Q3 & Q3 & Q3 \\
\hline Revista de Estudios Sociales & Colombia & Q4 & Q4 & Q4 & Q3 & Q3 & Q3 \\
\hline $\begin{array}{l}\text { Revista Española de } \\
\text { Investigaciones Sociológicas }\end{array}$ & España & Q3 & Q3 & Q2 & Q3 & Q3 & Q3 \\
\hline Clad & Venezuela & Q3 & Q3 & Q3 & Q4 & Q3 & Q3 \\
\hline Argos & Venezuela & Q4 & Q4 & Q4 & Q4 & Q4 & Q4 \\
\hline CEPAL Review & Chile & Q3 & Q4 & Q3 & Q4 & Q3 & Q3 \\
\hline
\end{tabular}

Fuente: elaboración propia a partir de datos de Scimago.

Presentado para evaluación: 1 de enero de 2018.

Aceptado para publicación: 8 de julio de 2018.

\section{SANTIAGO BASABE-SERRANO}

sbasabe@flacso.edu.ec

Coordinador del máster en Política Comparada y profesor titular del Departamento de Estudios Políticos en la Facultad Latinoamericana de Ciencias Sociales (FLACSO), sede de Ecuador. Sus áreas de investigación se concentran en las relaciones entre política y justicia, presidencialismo y estudios legislativos. Algunas de sus investigaciones recientes han sido publicadas en revistas académicas como Latin American Research Review, Political Research Quarterly, Journal of Latin American Studies, Justice System Journal, Revista de Ciencia Politica y Politica y Gobierno.

\section{SERGIO HUERTAS-HERNÁNDEZ}

sahuertasf@@lacso.edu.ec

Estudiante del máster en Política Comparada en la Facultad Latinoamérica de Ciencias Sociales (FLACSO), sede de Ecuador. Politólogo de la Universidad del Tolima (Colombia). Sus líneas de investigación son los estudios legislativos, las relaciones ejecutivo-legislativo y la historia y desarrollo de la ciencia política en América Latina. Pertenece al Grupo de Investigación sobre Historia de la Ciencia Política en América Latina (GIHCIPOLAL) de la Asociación Latinoamericana de Ciencia Política (ALACIP). 